\title{
DENOISING OF MEdiCAL IMAges USING IMAGE FUSION TECHNIQUES
}

\author{
V N Prudhvi Raj ${ }^{1}$ and Dr. T Venkateswarlu ${ }^{2}$ \\ ${ }^{1}$ Associate Professor, VR Siddhartha Engineering College Vijayawada, India \\ nagaprudhvi@yahoo.com \\ ${ }^{2}$ Professor, University College of Engineering, SV University, Tirupati, India
}

\begin{abstract}
Medical Imaging is playing the key role in diagnosing and treatment of diseases such as locating the tumours in brain, thin fractures in bones, detection of cancer cells in early stages etc. For making accurate decisions the images acquired by various medical imaging modalities must be free from noise. So Image denoising became an important pre-processing step in Medical image analysis. Developing the denoising algorithms is a difficult task because diagnostic information must be preserved while removing the noise. Earlier the denoising algorithms were designed in the spatial domain such as median filtering, harmonic filtering, and weiner filtering etc. by directly working on the pixel values, these methods will remove noise while introducing the blur in the denoised images. At present advanced mathematical models such as Partial differential equations which are useful for edge preservation and multiresolution analysis useful for preserving directional oriented information and texture became very popular in developing the denoising techniques. In this paper we are using Total Variational approach (PDE method) and Complex Dual Tree wavelet transform (Multiresolution analysis)method to denoise the medical images and we perform the fusion of the two denoised images resulting from the above denoising techniques. The performance of the proposed algorithm is compared with the existing methods using PSNR (Peak Signal to Noise Ratio) and SSIM (Structural Similarity Index.). The performance of the fusion process is measured using MI (Mutual Information), Edge Association and Spatial Frequency (SF) measures. The results showed that the proposed method is having better PSNR values diagnostically acceptable and very much useful for the diagnosis\& treatment phases.
\end{abstract}

\section{KEYWORDS}

Total Variation, Dual Tree Complex Wavelet Transform, SSIM, Spatial Frequency.

\section{INTRODUCTION}

Now a days Medical Informatics is providing the useful information of patients to the doctor in diagnosis and treatment phases. The medical informatics comprises of physiological signals such as ECG,EEG,EMG etc., Medical images such as Computed Tomography (CT), Magnetic Resonance Imaging (MRI), the different digital radiological processes for vascular, cardiovascular and contrast imaging, mammography, diagnostic ultrasound imaging, nuclear medical imaging with Single Photon Emission Computed Tomography (SPECT) and Positron Emission Tomography (PET) etc. as suggested by the physician. This information will be useful in analysing the patient physiological and anatomical condition to diagnose the diseases and to provide the treatment. The physiological signals and medical images will suffer from noise generated by the equipment in the acquisition phase and noise generated by the external sources

DOI : 10.5121/sipij.2012.3406 
Signal \& Image Processing : An International Journal (SIPIJ) Vol.3, No.4, August 2012

and transmission medium while transmitting from one place to another place as part of Telemedicine and Teleradiology. So the development of image denoising techniques became an active research area at present [1].

\section{Problem Formulation}

Let $f(x, y)$ be the object of interest (tissue or anatomical structures in the human body) and $g(x, y)$ be the image acquired by the imaging modality (CT, MRI, Ultrasound etc.) and corrupted by the noise $\eta(x, y)$. The imaging system will introduce certain amount of blur in the acquired image. The resultant image is suffering from two effects one is blurring and the other one is noise this phenomena is called image degradation. Now the goal is to design an algorithm to remove the blur and noise from the image. This process is called Image restoration process. When the removal of noise is alone considered the restoration process is called image denoising the following figure will summarise the image degradation and restoration process. In this paper we are considering noise alone so our aim is to reduce the noise effect and recover the image from its noisy version without losing the diagnostically relevant information[1].

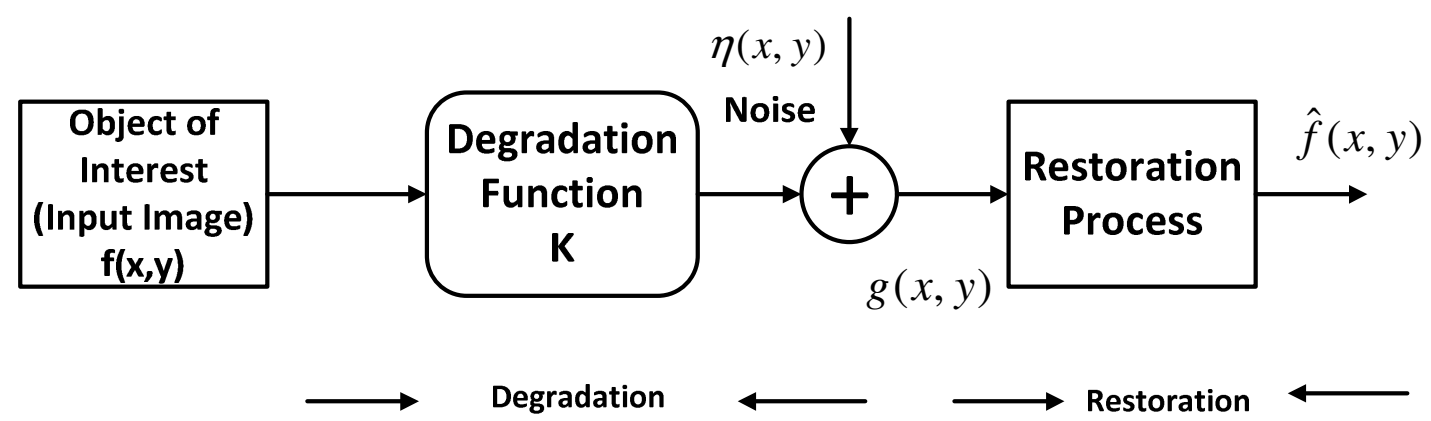

Figure 1: Image Degradation and Restoration Process

Spatial filters are traditional means of removing noise from images and signals. Spatial filters usually smooth the data to reduce the noise, and also blur the data. Several new techniques have been developed in the last few years that improve on spatial filters by removing the noise more effectively while preserving the edges in the data. Some of these techniques used the ideas from partial differential equations and computational fluid dynamics such as level set methods, total variation methods [3], non-linear isotropic and anisotropic diffusion, other techniques combine impulse removal filters with local adaptive filtering in the transform domain to remove not only white and mixed noise, but also their mixtures. In order to reduce the noise present in medical images many techniques are available like digital filters (FIR or IIR), adaptive filtering methods etc. However, digital filters and adaptive methods can be applied to signal whose statistical characteristics are stationary in many cases $[1,3]$. Recently the wavelet transform has been proven to be useful tool for non-stationary signal analysis. Many denoising algorithms were developed on wavelet framework effectively but they suffer from four shortcomings such as oscillations, shift variance, aliasing, and lack of directionality. In this paper we will present total variational approach and denoising using DTCWT in sections 3 and 4 and the fusion process in section 5.sections 6 will present the results and section 7 concludes the paper.

\section{TOTAL VARIATIONAL APPROACH}

Since the last decade in signal and image processing areas, denoising of signals and images using Total variation approach, which is well known as total variation regularization is gained the 
popularity because of its edge preserving nature while performing the denoising compared to the other traditional spatial filtering approaches[2]. It is observed that the noise will be at high frequencies and the signals and images with excessive \& spurious detail will have the high total variation i.e. the integral of the absolute gradient of those signals and images is high. Based on these observations it is proposed to reduce the total variation of the signal or image subject to it for getting a close match to the original signal. This concept was pioneered by Rudin et al. in 1992.For a digital signal $y_{n}$, the total variation is defined as:

$$
V(y)=\sum_{n}\left|y_{n+1}-y_{n}\right|
$$

Given an input signal $x_{n}$, the aim of total variation method is to find an approximation signal call it, $y_{n}$, which is having smaller total variation than $x_{n}$ but is "close" to $x_{n}$. One of the measures of closeness is the sum of square errors:

$$
E(x, y)=\frac{1}{2} \sum_{n}\left(x_{n}-y_{n}\right)^{2}
$$

So the total variation approach achieves the denoising by minimizing the following discrete functional over the signal $y_{n}$ :

$$
E(x, y)+\lambda V(y)
$$

By differentiating the above functional with respect to $y_{n}$, in the original approach we will derive a corresponding Euler-Lagrange equation which is numerically integrated with $x_{n}$ (the original signal) as initial condition. Since this problem is a convex functional, we can use the convex optimization techniques to minimize it to find the solution $y_{n}$. In this paper we used the splitBregman iterative method to solve the above problem and to get the denoised image.

\section{Complex Dual Tree Wavelet Transform}

\subsection{Discrete Wavelet Transform}

The DWT of a signal $x(n)$ is calculated by passing it through a series of filters. First the samples are passed through a low pass filter with impulse response $h_{0}(n)$ resulting in a convolution of the two:

$$
y[n]=\left(x * h_{0}\right)[n]=\sum_{k=-\infty}^{\infty} x(k) h_{0}(n-k)
$$

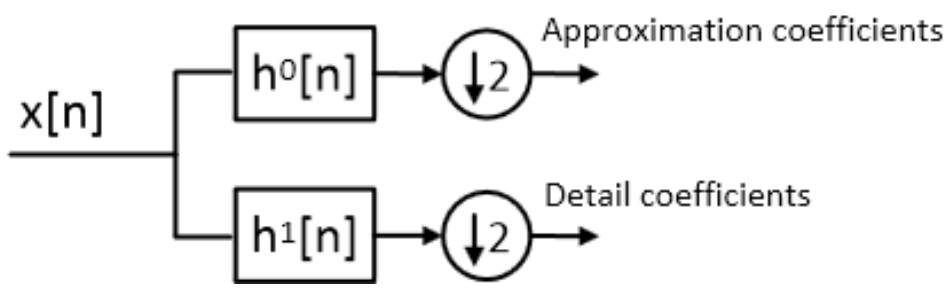

Figure 2: DWT Single stage Decomposition 
The signal is also decomposed simultaneously using a high-pass filter $h_{1}(n)$. The outputs of the highpass filter are detail coefficients and the outputs of the lowpass filter are approximation coefficients. It is important that the two filters are related to each other and they are known as a quadrature mirror filter. Since half the frequencies of the signal have now been removed, half the samples can be discarded according to Nyquist's rule. The filter outputs are then subsampled by $2[9,11]$.

$$
\begin{aligned}
& y_{\text {low }}[n]=\sum_{k=-\infty}^{\infty} x(k) h_{0}(2 n-k) \\
& y_{\text {high }}[n]=\sum_{k=-\infty}^{\infty} x(k) h_{1}(2 n+1-k)
\end{aligned}
$$

This decomposition has halved the time resolution since only half of each filter output characterises the signal. However, each output has half the frequency band of the input so the frequency resolution has been doubled.

This decomposition is repeated to further increase the frequency resolution and the approximation coefficients decomposed with high and low pass filters and then down-sampled. This is represented as a binary tree with nodes representing a sub-space with a different time-frequency localisation. The tree is known as a filter bank.

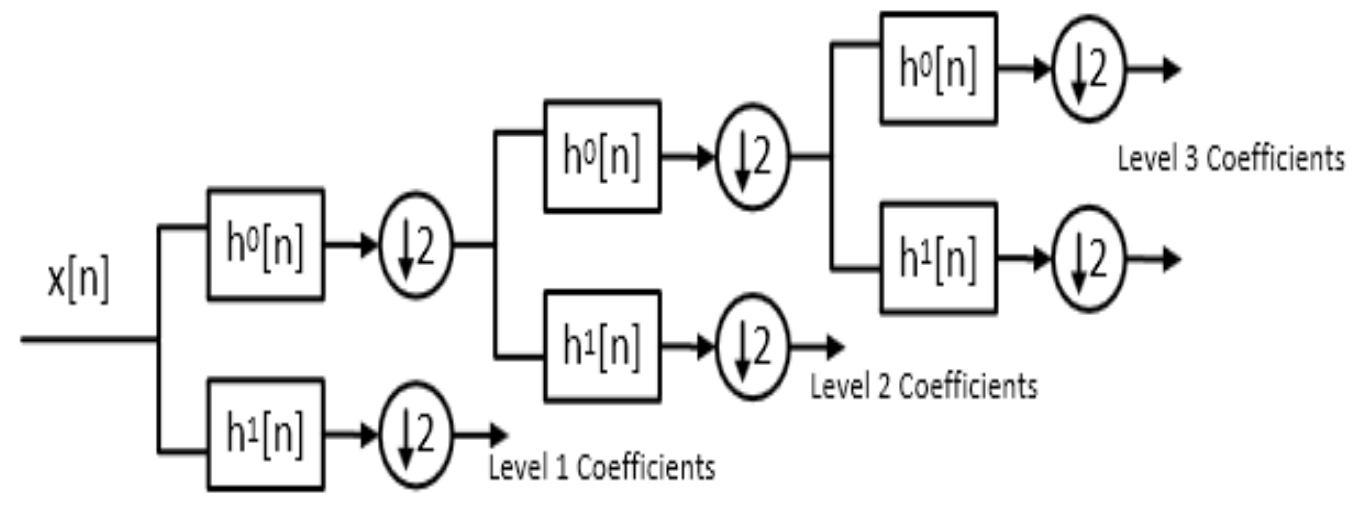

Figure 3: DWT Multilevel Decomposition

2D DWT can be implemented by applying the 1D DWT along the rows of an image first and applying then on the columns of an image. When a wavelet transform is applied to an image which is a $2 \mathrm{D}$ signal it decomposes the image into four subbands as shown in the following figure. The LL band contains the approximation coefficients, LH band contains horizontal details, HL band contains vertical details and $\mathrm{HH}$ band will contain the diagonal details [9]. 
Signal \& Image Processing : An International Journal (SIPIJ) Vol.3, No.4, August 2012

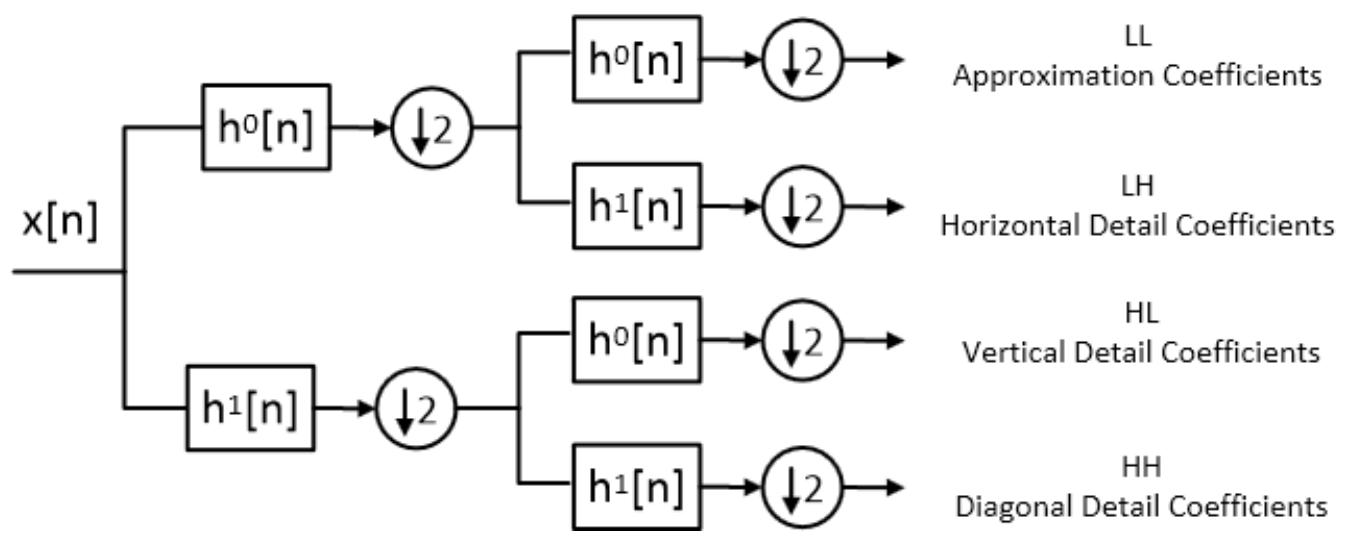

Figure 4: 2D DWT Single stage Decomposition

\section{Advantages of DWT}

$>$ The ability to compact most of the signals energy into a few transformation coefficients which is called energy compaction.

$>$ The ability to capture and represent effectively low frequency components as well as high frequency transients.

$>$ The variable resolution decomposition with almost uncorrelated coefficients.

\subsection{Dual Tree Complex Wavelet Transform}

The dual tree complex wavelet transform is directionally selective and shift invariant in two and higher dimensions. The dual tree complex wavelet transform introduces the redundancy by a factor of $2^{d}$ for $d$-dimensions which is lower than the redundancy introduced by UDWT (Undecimated Wavelet Transform). Since last 20 years DWT (Discrete Wavelet Transform) has proven excellent tool for analysis of one dimensional signal's by replacing the Fourier Transform's infinitely oscillating sinusoidal basis functions with a set of locally oscillating functions called wavelets. But its performance is poor in the analysis of complex and modulated signals such as radar, speech, music, higher dimensional medical and geophysics data. In these areas the complex wavelet transform will give a better performance than critically sampled DWT. The Discrete wavelet transform is suffering from four shortcomings.

Oscillations: The wavelets are bandpass functions, so the coefficients of wavelet transform will oscillate positive and negative around singularities. This will complicate signal modelling and singularity extraction.

Shift Variance: A Small shift in the signal of interest will perturb the wavelet coefficient oscillation pattern around singularities. The wavelet transform coefficients of signal $x(t)$ and its shifted version $\mathrm{x}(\mathrm{t}-\mathrm{d})$ are not same.

Aliasing: The wavelet coefficient processing methods like thresholding, filtering and quantization etc will upset the balance between the Analysis and synthesis filter banks so the artifacts will present in the reconstructed signal.

Lack of Directionality: DWT will process the point singularities effectively but it can be difficult to model and process the geometric features like lines, edges and ridges. 
These four short comings can be minimised by using dual tree wavelet transform in processing the medical images than the traditional DWT and UDWT. UDWT is shift invariant but the redundancy introduced is more than the dual tree wavelet transforms. There are three wavelets associated with the 2D wavelet transform. The following figure illustrates three wavelets as gray scale images.

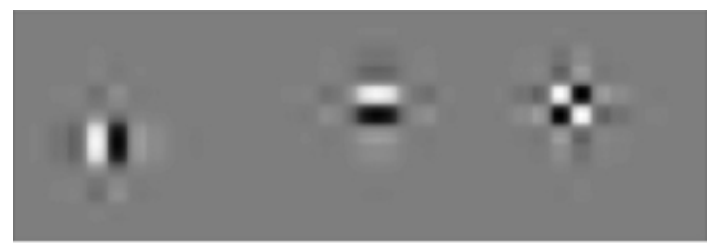

Figure 5: 2D Wavelets

The first two wavelets are oriented in the vertical and horizontal directions and the third wavelet does not have a dominant orientation. The third wavelet mixes two diagonal orientations, which gives rise to the checkerboard artifact. The 2D DWT is poor at isolating the two diagonal orientations i.e it can't distinguish $+45^{\circ}$ line and $-45^{\circ}$. The complex 2-D dual-tree DWT have wavelets in six distinct directions as shown in the following figure. There are two wavelets in each direction. In each direction, one of the two wavelets can be interpreted as the real part of a complex-valued 2D wavelet, and the other wavelet as the imaginary part of a complex-valued 2D wavelet.

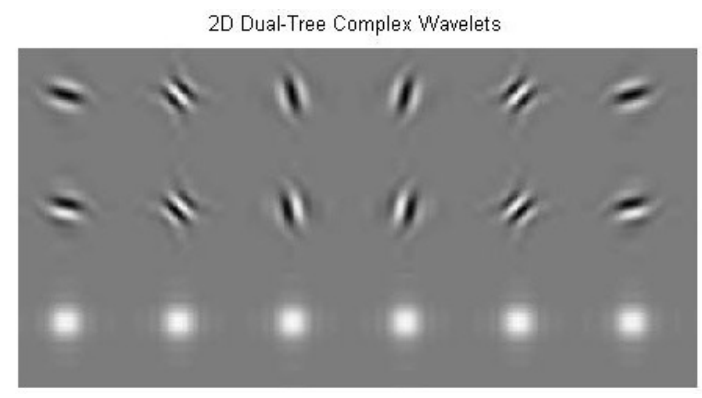

Figure 6: 2D Dual Tree Complex Wavelets

The dual-tree complex DWT of a signal $\mathrm{x}$ is computed using two critically-sampled DWTs in parallel on the same data as shown in the following figure. If the same filters used in the upper tree and lower tree nothing is gained. So the filters in this structure will designed in a specific way that the subbands of upper DWT is interpreted as real part of complex wavelet transform and the lower tree as imaginary part. The transform is expansive by a factor 2 and shift invariant. 


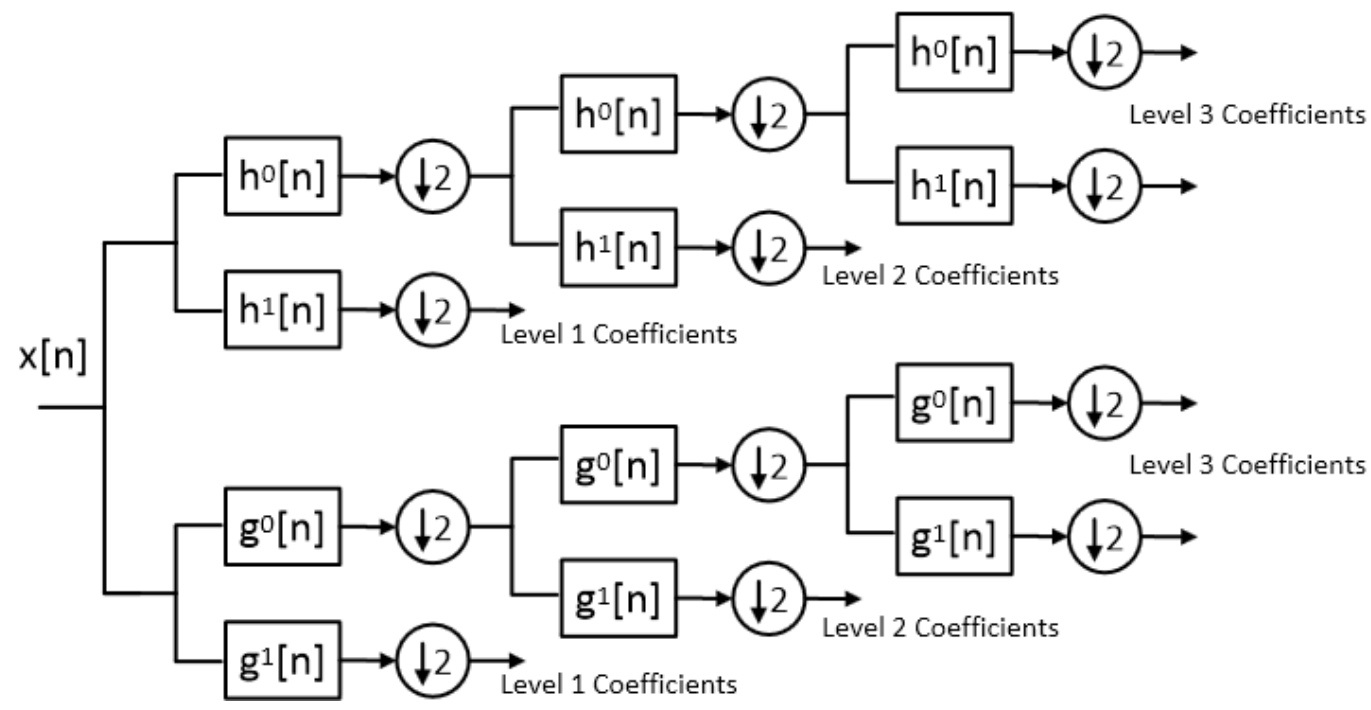

Figure 7: Dual Tree Complex Wavelet Filter bank

The complex 1D wavelet is shown in the following figure

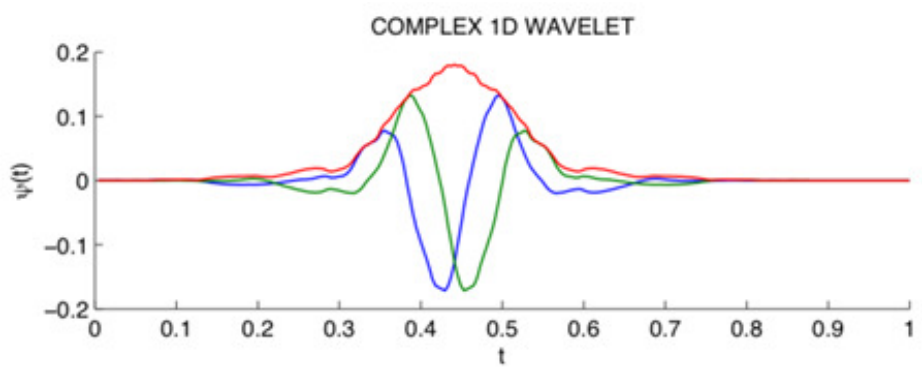

Figure 8: 1D Dual Tree Complex Wavelet

There are various methods to design the filters for dual tree complex wavelet transform. The detailed study of filter design is found in the article "The Dual-Tree Complex Wavelet Transform" by Nick G. Kingsbury [12]. The filters must satisfy the desired properties such as approximate half sample property, Perfect Reconstruction (Orthogonal or Biorthogonal), Finite support (FIR filters), and Vanishing moments/good stop band, Linear phase.

\subsection{Denoising using Dual Tree Complex Wavelet Transform}

The various steps involved in denoising of images using wavelets is shown in the following block diagram

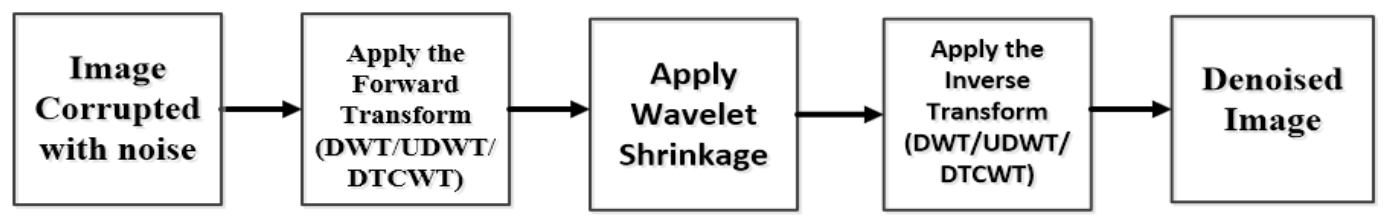

Figure 9: Block diagram for Denoising of images using wavelet Shrinkage 
Signal \& Image Processing : An International Journal (SIPIJ) Vol.3, No.4, August 2012

Step1: Decompose the given image into subbands using the forward wavelet transforms like DWT (Discrete Wavelet Transform), UDWT (Undecimated Discrete Wavelet Transform), and DTCWT (Dual Tree Complex Wavelet Transform). In this paper we used the Dual Tree complex wavelet transform for the decomposition.

Step 2: Calculate the Threshold globally or adaptively (Level by Level or Subband by Subband) and Threshold the wavelet coefficients using Hard Thresholding, Soft Thresholding or Semi soft thresholding. In this paper we used the subband dependent threshold along with soft thresholding called as Bayes shrink $[6,7,8]$.

Step 3: Apply the Inverse Transform to reconstruct the denoised image from modified wavelet coefficients.

\section{Proposed METHOD}

Image fusion is the process of integrating two or more images to form one image which is highly informative. The main aim of this process is to extract the features which are perceptually important from all the images and combine them to form a fused image which is more suitable for computer processing and human visual perception. Image fusion is playing a major role in the research areas such as Medical Imaging, Remote sensing, computer vision, Robotics and Microscopic imaging etc [13].

In this paper we are proposing the new method which uses the two famous denoising procedures i.e. Total variational approach and Dual tree complex wavelet transform to reduce the noise and fuse the results using the fusion techniques to combine the benefits of both denoising algorithms. The resultant image will have optimum edge preservation and texture which are very much required in the diagnosis and treatment phases. The experimental setup for the above procedure is shown below.

Sharp images contain more details than blurred images. In many situations great variations are present in scene's depth; it is difficult to acquire an image in which all the areas of scene appear sharp. The scene areas which are in-focus will appear sharp and has higher contrast. Areas which are out of focus i.e. in front of or behind the focus plane will be blurred. So the key challenge of image fusion process is to identify the relevant information such as contrast/ high local energy and areas that are having high sharpness from the best-focused images and combine this useful information to create a highly informative image.

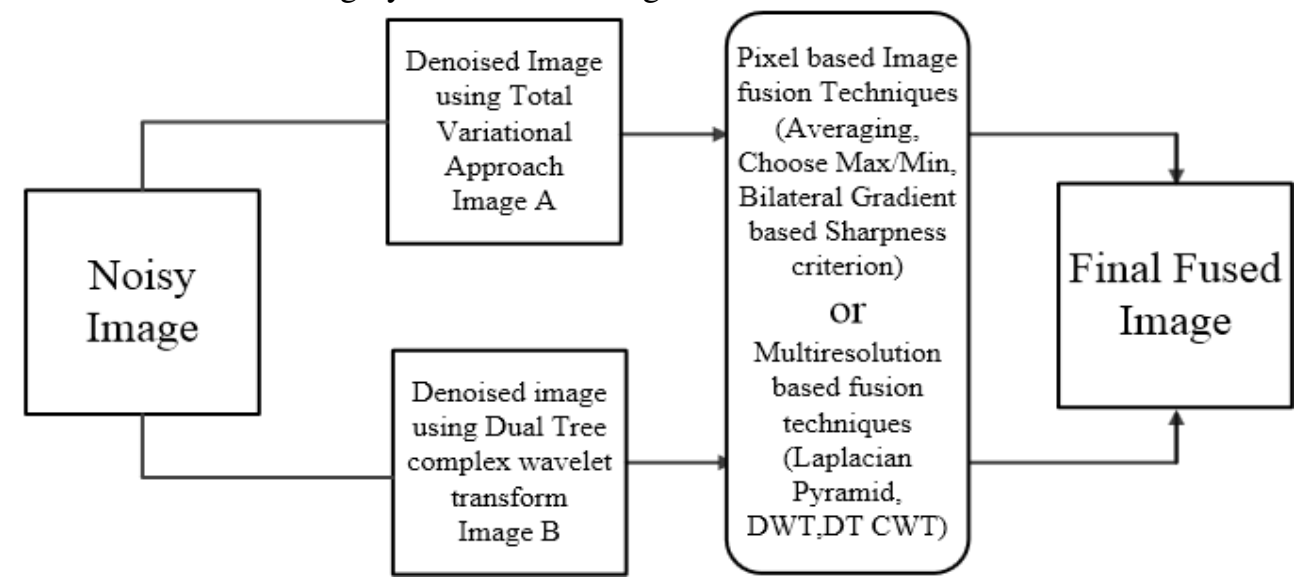

Figure 10: Block Diagram of Denoising using Fusion Techniques 
Signal \& Image Processing : An International Journal (SIPIJ) Vol.3, No.4, August 2012

In this paper we are using pixel based fusion techniques and multiresolution transform techniques while measuring sharpness of the image. An image with higher phase coherence and higher strength is considered as sharper and more informative.

Given a set of 2-D images $I_{1}(x, y), I_{2}(x, y), \ldots \ldots, I_{n}(x, y)$ which are captured with different imaging settings in the camera and aligned well, the aim of multi-focus image fusion is to combine the information content of the individual images into a single fused image $f(x, y)$. Normalized aggregation of the images is a simple method for image fusion. This can be expressed as

$$
f(x, y)=\frac{1}{N} \sum_{n=1}^{N} I_{n}(x, y)
$$

The main drawback with this method is that all information content within the images is given equal importance. Therefore, image regions such as edges and boundaries which are more informative, are treated no differently than unimportant regions. A normalized weighted aggregation approach was introduced to overcome this situation. It can be mathematically expressed as

$$
f(x, y)=\frac{\sum_{n=1}^{N} w_{n}(x, y) I_{n}(x, y)}{\sum_{n=1}^{N} w_{n}(x, y)}
$$

where $w_{n}(x, y)$ is the weight assigned to information content at $(x, y)$ in the $\mathrm{n}$-th image. The choice of the weighting scheme (i.e., the determination of $w_{n}(x, y)$ in Eq. (7) is important in the performance of the image fusion algorithm which is required to be locally adaptive to image's content and be sensitive to the blur of each image. In view of this, a brief survey on existing sharpness measures will be discussed here.

\subsection{Existing Sharpness Criterion}

To measure the degree of images blur the high-frequency information such as edges and boundaries are usually used as the basis. In general sharper edges are present in the well-focused images and is expected to have higher frequency content than those that are blurred. In the following analysis, denote $I(x, y)$ be the intensity value at the position $(x, y)$ of the image $\mathrm{I}$.

Variance [14]. For an $M \times N$ block of the image, its variance is defined as

$$
S_{V A R}=\frac{1}{M \times N} \sum_{x} \sum_{y}(I(x, y)-\mu)^{2}
$$

Where $\mu$ is the mean intensity value of the image block and it is defined as

$$
\mu=\frac{1}{M \times N} \sum_{x} \sum_{y}(I(x, y))
$$


Energy of image gradient [14]. For a $M \times N$ block of the image, it is measured as

$$
S_{E G}=\sum_{x} \sum_{y}\left(I_{x}^{2}+I_{y}^{2}\right)
$$

where $I_{x}$ and $I_{y}$ represent image gradients at the row and column directions, respectively. They are usually defined as $I_{x}=I(x+1, y)-I(x, y)$ and $I_{y}=I(x, y+1)-I(x, y)$.

Tenenbaum. For an block of the image, it is measured as

$$
S_{T N G}=\sum_{x} \sum_{y}(\nabla I(x, y))^{2}
$$

Where $\nabla I(x, y)=\sqrt{I_{x}{ }^{2}+I_{y}{ }^{2}}$ in which $I_{x}$ and $I_{y}$ are gradients (obtained using Sobel operators) along the row and column directions, respectively.

Energy of Laplacian [14]. For an $M \times N$ block of the image, it is measured as

$$
S_{E L}=\sum_{x} \sum_{y}\left(\nabla^{2} I(x, y)\right)^{2}
$$

where $\nabla^{2} I(r, c)$ represents image gradient obtained by Laplacian operator $[-1,-4,-1 ;-4$, $20,-4 ;-1,-4,-1]$.

Sum-modified-Laplacian [14]. It differs from the usual Laplacian operator in that the absolute values of the partial second derivatives are summed instead of their actual values. That is, it is mathematically expressed as

$$
S_{S M L}=\sum_{x} \sum_{y}\left(\nabla^{2} I(x, y)\right)^{2}
$$

Where $\nabla^{2} I(x, y)=|2 I(x, y)-I(x+1, y)-I(x-1, y)|+|2 I(x, y)-I(x, y+1)-I(x, y-1)|$.

Frequency selective weighted median filter [14]. It measures the sharpness of the image as

$$
S_{F S W M}=\sum_{x} \sum_{y}\left(I_{x}^{2}+I_{y}^{2}\right)
$$

Where

$$
\begin{aligned}
I_{y}= & \operatorname{med}\{I(x, y-1), I(x, y), I(x, y+1)\}-\frac{1}{2} \operatorname{med}\{I(x, y-3), I(x, y-2), I(x, y-1)\} \\
& -\frac{1}{2} \operatorname{med}\{I(x, y+1), I(x, y+2), I(x, y+3)\} . \\
I_{x}= & \operatorname{med}\{I(x-1, y), I(x, y), I(x+1, y)\}-\frac{1}{2} \operatorname{med}\{I(x-3, y), I(x-2, y), I(x-1, y)\} \\
& -\frac{1}{2} \operatorname{med}\{I(x+1, y), I(x+2, y), I(x+3, y)\},
\end{aligned}
$$


Phase coherence model: It is consistent to the perceptual significance of the image [15, 17, 22] and it can be determined at a particular position (x,y) as

$$
S_{P C M}(x, y)=\frac{1}{2} \sum_{\theta}\left|(h(x, y, \theta) \sin (\theta))^{2}+(h(x, y, \theta) \cos (\theta))^{2}\right|+\frac{1}{2} \sqrt{4 K^{2}+L^{2}}
$$

Where

$$
\begin{aligned}
& K=\sum_{\theta}(h(x, y, \theta) \sin (\theta) h(x, y, \theta) \cos (\theta))^{2} \\
& L=\sum_{\theta}\left[(h(x, y, \theta) \cos (\theta))^{2}-(h(x, y, \theta) \sin (\theta))^{2}\right], \\
& h(x, y, \theta)=\frac{\sum_{n} w(x, y, \theta)\left|A_{n}(x, y, \theta) \nabla \varphi_{n}(x, y, \theta)\right|}{\sum_{n} A_{n}(x, y, \theta)+\xi}, \\
& \Delta \varphi_{n}(x, y, \theta)=\cos \left(\varphi_{n}(x, y, \theta)-\bar{\varphi}_{n}(x, y, \theta)-\left|\sin \left(\varphi_{n}(x, y, \theta)-\bar{\varphi}_{n}(x, y, \theta)\right)\right|\right.
\end{aligned}
$$

in which $\mathrm{W}$ represents the frequency spread weighting factor, $A_{n}$ and $\varphi_{n}$ represent the amplitude and phase at the wavelet scale n, respectively, $\bar{\varphi}_{n}$ represents the weighted mean phase, $\xi$ is a small constant used to avoid the division by zero. All of these parameters are as same as that used in.

\section{Bilateral Gradient based Sharpness Criterion}

The statistics of image's gradient is examined in this section to propose a new sharpness measurement criterion, which exploits bilateral statistics of image's gradient information [21]. Image structure can be measured effectively by using the image gradients. Consider an image of interest $I(x, y)$. The gradient covariance matrix of a region within an $M \times N$ local window is defined as

$$
C=\left(\begin{array}{cc}
\sum_{w} I_{x}^{2}(x, y) & \sum_{w} I_{x}(x, y) I_{y}(x, y) \\
\sum_{w} I_{x}(x, y) I_{y}(x, y) & \sum_{w} I_{y}^{2}(x, y)
\end{array}\right)
$$

Where $I_{x}(x, y)$ and $I_{y}(x, y)$ represent image's gradient at the row and column directions, respectively. Furthermore, the above gradient covariance matrix can be decomposed as

$$
C=V D V^{T}=\left(\begin{array}{ll}
v_{1} & \mathrm{v}_{2}
\end{array}\right)\left(\begin{array}{cc}
\lambda_{1} & 0 \\
0 & \lambda_{2}
\end{array}\right)\left(\begin{array}{l}
v_{1}^{T} \\
v_{2}^{T}
\end{array}\right)
$$

where $\mathrm{V}$ represents a $2 \times 2$ matrix whose column vectors are eigenvectors $v_{1}$ and $v_{2}$, D denotes the $2 \times 2$ diagonal matrix whose diagonal elements are eigenvalues $\lambda_{1}$ and $\lambda_{2}\left(\lambda_{1} \succ \lambda_{2}\right)$ that correspond to eigenvectors $\mathrm{v} 1$ and $\mathrm{v} 2$, respectively, and the superscript $\mathrm{T}$ denotes the transpose.

The geometrical structure at a pixel in an image can be described by the eigenvalues $\lambda 1$ and $\lambda 2$ of the above gradient covariance matrix. Motivated by this, the first criterion is proposed to measure the strength of the image's gradient, which is defined as

$$
A(x, y)=\lambda_{1}-\lambda_{2}
$$


Signal \& Image Processing : An International Journal (SIPIJ) Vol.3, No.4, August 2012

On the other hand, local phase coherence is consistent with the perceptual significance of image's characteristics. This has been supported by the physiological evidence that showed high human perception response to signal characteristics with high local phase coherence. Another advantage is the fact that it is insensitive magnitude variations caused by illumination conditions or noise incurred in image signals. In view of this, the second criterion is to consider the phase coherence of the image's gradient, that is

$$
P(x, y)=-\cos (\theta(x, y)-\bar{\theta}(x, y))
$$

where $\theta(x, y)$ is the phase information at the position $(x, y)$ determined by the principle Eigen

vector $v_{1}$ associated with the largest eigenvalue $\lambda_{1}$ defined as (13), $\bar{\theta}_{x, y}$ is the average of phases of the neighbouring positions. This measure achieves the maximal value when the local phase coherence is worst, which is usually caused by an edge. Finally, the above two criterions (22) and (23) are jointly considered to develop a bilateral sharpness criterion as

$$
S_{B S C}=A^{\alpha}(x, y) P^{\beta}(x, y)
$$

Where $\alpha$ and $\beta$ are two factors to adjust contributions of two criterions.

\subsection{Fusion Process}

\section{Pixel based Fusion process}

Step 1: Verify all the Images are of same size or not if not reject the fusion process.

Step 2: Decompose the Images into multiple blocks.

Step 3: Find the Energy and sharpness of the each block using the above sharpness criterions.

Step 4: Perform the fusion process by choosing the block having high energy and sharpness measure from the two images.

Step 5: Reconstruct the image from the resultant Intermediate blocks to get the fused image.

The following block diagram will give you the steps of fusion process. 
Signal \& Image Processing : An International Journal (SIPIJ) Vol.3, No.4, August 2012

Decomposed Image A

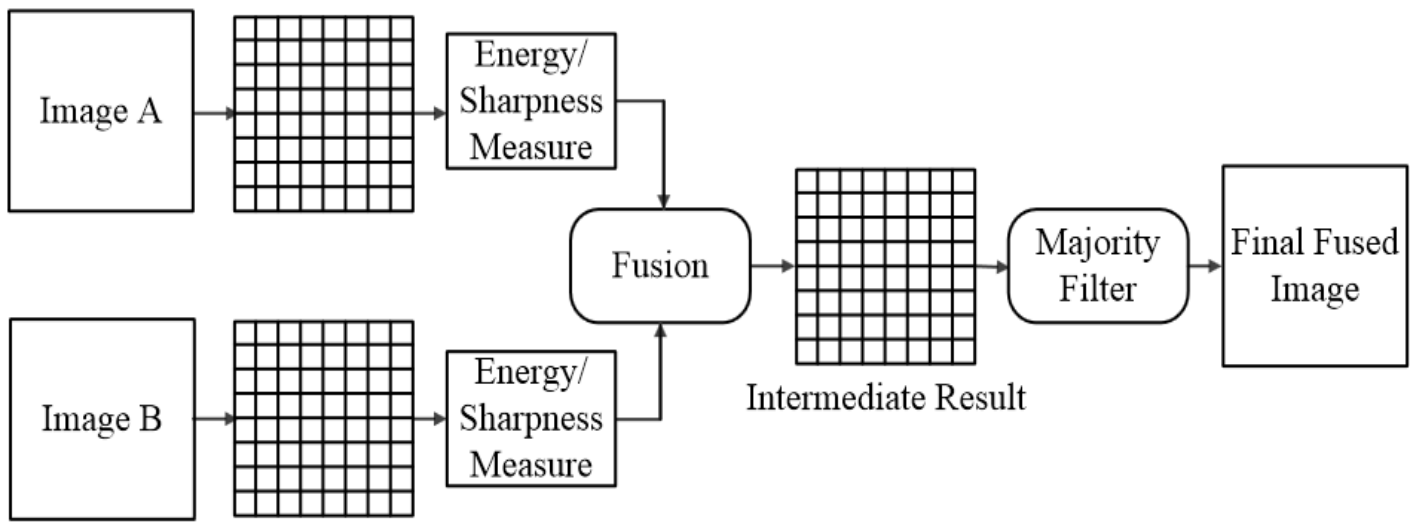

Decomposed Image B

Figure 11: Block diagram of Pixel level Fusion process technique

\section{Multiresolution based Fusion process}

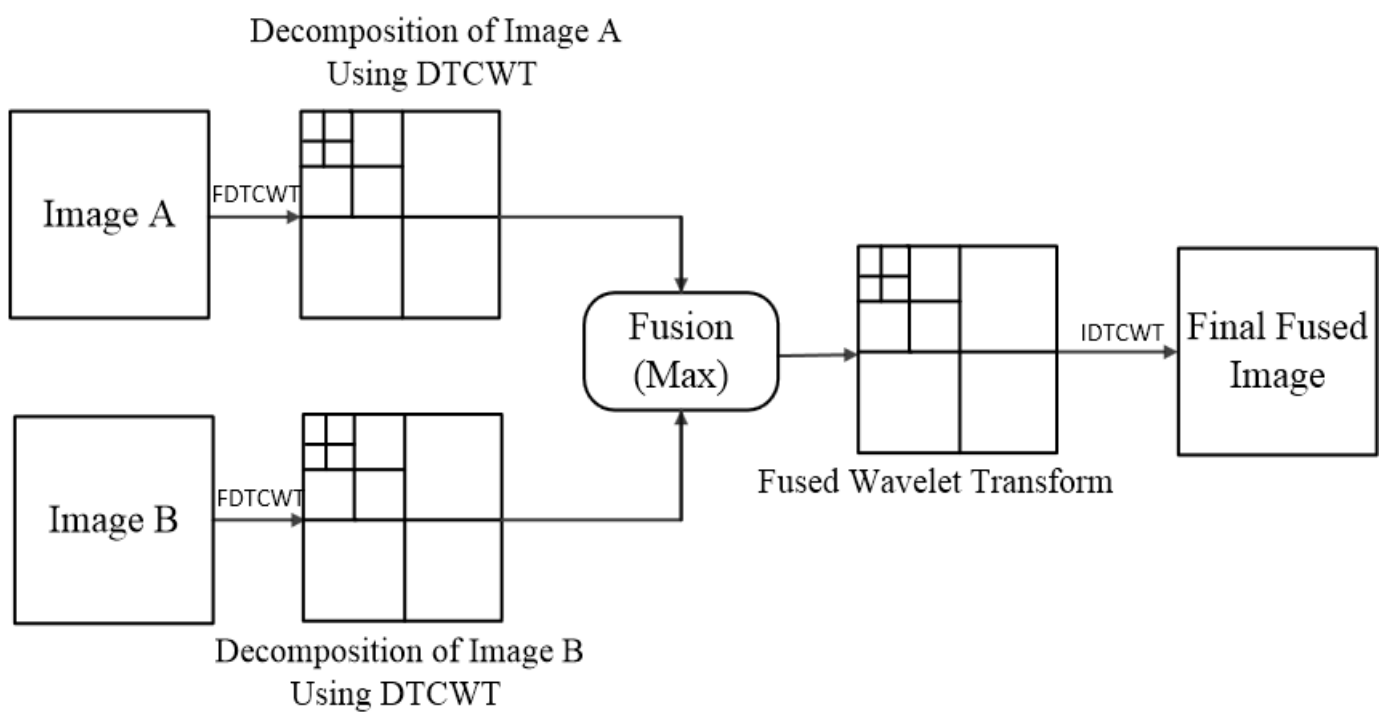

Figure 12: Block diagram of Multiresolution based Fusion process technique

Step 1: Verify all the Images are of same size or not if not reject the fusion process.

Step 2: Apply the Forward Multiresolution Transform (Laplacian Pyramid, DWT, UDWT, and Dual Tree Complex Wavelet Transform) to decompose the Images into subbands.

Step 3: Find the Energy and sharpness of the each subband using the above sharpness criterions.

Step 4: Perform the fusion process by choosing the subband having high energy and sharpness measure from the two images. 
Signal \& Image Processing : An International Journal (SIPIJ) Vol.3, No.4, August 2012

Step 5: Reconstruct the image from the resultant Intermediate subbands by applying inverse transform to get the fused image.

The fusion methods using wavelet transforms can be found in [16, 18, 19, and 20].

The following flowchart will give you the steps of fusion process.

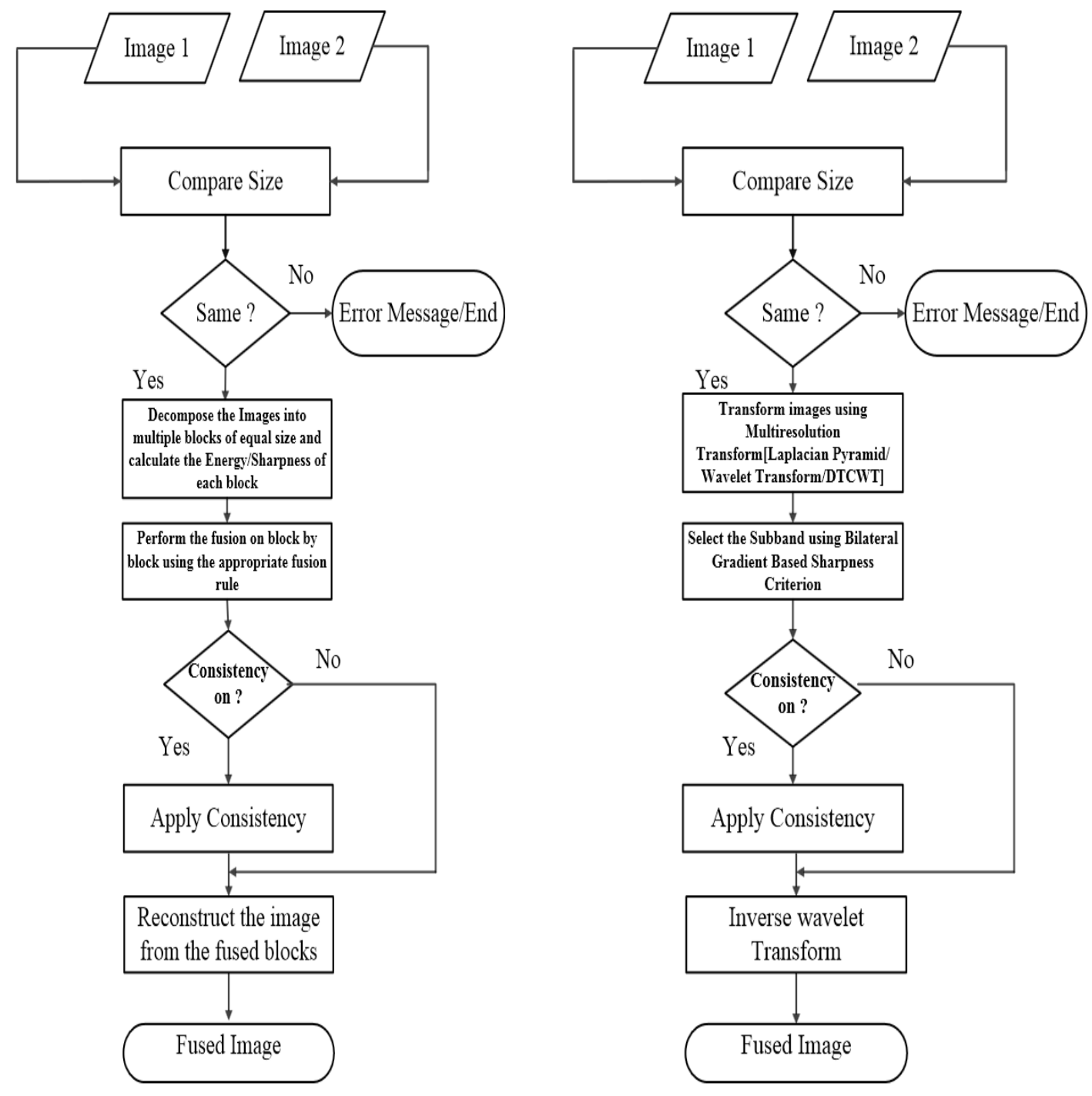

Figure 13: Flowcharts of pixel level and Multiresolution based Fusion process technique

\section{RESUlts}

The images corrupted with Gaussian noise are first denoised using total variational approach and Dual Tree complex wavelet transform then these results are fused using various fusion techniques to improve the quality of the image by combining the best features from the denoised images.

The efficiency of denoising the images is evaluated using PSNR (Peak Signal to Noise Ratio) and SSIM (Structural Similarity Index measure) measures.

\section{PSNR (Peak Signal to Noise Ratio)}


PSNR is the peak signal-to-noise ratio in decibels (dB). The PSNR is only meaningful for data encoded in terms of bits per sample, or bits per pixel. For example, an image with 8 bits per pixel contains integers from 0 to 255 .

$$
P S N R=20 \log _{10}\left(\frac{2^{B}-1}{\sqrt{M S E}}\right)
$$

Where B represents bits per sample and

MSE (Mean Squared error) is the mean square error between a signal $f(x, y)$ and an approximation $\hat{f}(x, y)$ is the squared norm of the difference divided by the number of elements in the signal.

$$
M S E=\|f(x, y)-\hat{f}(x, y)\|^{2}=\frac{1}{M N} \sum_{x=0}^{M} \sum_{y=0}^{N}[f(x, y)-\hat{f}(x, y)]^{2}
$$

Structural similarity (SSIM) index is a method for measuring the similarity between two images [10]. The SSIM index is a full reference metric, in other words, the measuring of image quality based on an initial uncompressed or distortion-free image as reference. SSIM is designed to improve on traditional methods like peak signal-to-noise ratio (PSNR) and mean squared error (MSE), which have proved to be inconsistent with human eye perception [10].

The SSIM metric is calculated on various windows of an image. The measure between two windows $x$ and $y$ of common size $N \times N$ is [10]:

$$
\operatorname{SSIM}(x, y)=\frac{\left(2 \mu_{x} \mu_{y}+c_{1}\right)\left(2 \sigma_{x y}+c_{2}\right)}{\left(\mu_{x}^{2}+\mu_{y}^{2}+c_{1}\right)\left(\sigma_{x}^{2}+\sigma_{y}^{2}+c_{2}\right)}
$$

Where

$>\mu_{x}$ is the average of $x$ and $\mu_{y}$ is the average of $y$

$>\sigma_{x}^{2}$ is the variance of $x$ and $\sigma_{y}^{2}$ is the variance of $y$

$>\sigma_{x y}$ is the covariance of $x$ and $y$

$>c_{1}=\left(k_{1} L\right)^{2}, c_{2}=\left(k_{2} L\right)^{2}$ two variables to stabilize the division with weak denominator

$>L$ is the dynamic range of the pixel values (typically this is $2^{\# \text { bits per pixel }}-1$ )

$>k_{1}=0.01$ and $k_{2}=0.03$ by default

In order to evaluate the image quality this formula is applied only on luma. The resultant SSIM index is a decimal value between -1 and 1 , and value 1 is only reachable in the case of two identical sets of data. Typically it is calculated on window sizes of $8 \times 8$. The window can be displaced pixel-by-pixel on the image.

To evaluate the performance of the fusion process in this paper we used the Mutual Information (MI), edge-association $\left(\mathrm{Q}^{\mathrm{AB} / \mathrm{F}}\right)$, spatial frequency $(\mathrm{SF})$ along with the RMSE (Root Mean Square error) and STD (Standard Deviation). The comparison of the proposed algorithm was carried out with the existing Bilateral Gradient based Sharpness criterion, Laplacian Pyramid method and Image fusion using DWT. 
RMSE:

RMSE will measure the difference between the ideal image and the fused image. It is defined as

$$
R M S E=\sqrt{\frac{1}{M N} \sum_{x=0}^{M} \sum_{y=0}^{N}[g(x, y)-f(x, y)]^{2}}
$$

\section{Spatial Frequency (SF):}

Spatial frequency is defined as [26]

$$
S F=\sqrt{R F^{2}+C F^{2}}
$$

Where RF and CF are the row frequency

$$
R F=\sqrt{\frac{1}{M N} \sum_{x=0}^{M-1} \sum_{y=0}^{N-1}[f(x, y)-f(x, y-1)]^{2}}
$$

and column frequency

$$
C F=\sqrt{\frac{1}{M N} \sum_{x=0}^{M-1} \sum_{y=0}^{N-1}[f(x, y)-f(x-1, y)]^{2}}
$$

Respectively and $f(x, y)$ is the fused image.

\section{Mutual Information (MI)}

It is well known that MI is a basic concept of information theory measuring the statistical dependence between two random variables and the amount of information that one variable contains about the others. The measure presented here states that the MI describes the similarity of the image intensity distributions of the corresponding image pair [24].

Let $\boldsymbol{A}$ and $B$ be two random variables with marginal probability distributions $p_{A}(a)$ and $p_{B}(b)$ and joint probability distribution $p_{A B}(a, b)$. MI measures the degree of dependence of the two random variables $\boldsymbol{A}$ and $B$. It is defined by Kullback-Leibler measure

$$
I_{A B}(a, b)=\sum_{x, y} p_{A B}(a, b) \log \frac{p_{A B}(a, b)}{p_{A}(a) p_{B}(b)}
$$

The fused image should of course contain the important information from the original image sets. Obviously the notion of 'important information' depends on the application and is difficult to define. Mutual information is the amount of information that one image contains about another.

Considering two input images $\mathrm{A}, \mathrm{B}$ and a fused image $\mathrm{F}$ we can calculate the amount of information that $\mathrm{F}$ contains about $\mathrm{A}$ and $\mathrm{B}$ according to above equation

$$
\begin{aligned}
& I_{F A}(f, a)=\sum_{x, y} p_{F A}(f, a) \log \frac{p_{F A}(f, a)}{p_{F}(f) p_{A}(a)} \\
& I_{F B}(f, b)=\sum_{x, y} p_{F B}(f, b) \log \frac{p_{F B}(f, b)}{p_{F}(f) p_{B}(b)}
\end{aligned}
$$

Thus the mutual information is given as 


$$
M_{F}^{A B}=I_{F A}(f, a)+I_{F B}(f, b)
$$

The above equation indicates that the total information that a fused image $\mathrm{F}$ contain about $\mathrm{A}$ and B.

\section{Edge Association}

In this method an important visual information with the "edge" information that is present in each pixel of an image. Notice that this visual to edge information association is supported by Human Visual System studies and is extensively used in image analysis and compression systems [24, 26].

The amount of edge information that is transferred from input images to the fused image can be obtained as

$$
Q_{p}^{A B / F}=\frac{\sum_{x=1}^{M} \sum_{y=1}^{N} Q^{A F}(x, y) w^{A}(x, y)+Q^{B F}(x, y) w^{B}(x, y)}{\sum_{x=1}^{M} \sum_{y=1}^{N}\left(w^{A}(x, y)+w^{B}(x, y)\right)}
$$

Where $Q^{A F}, Q^{B F}$ are edge preservation values and $w^{A}, w^{B}$ are the corresponding weights

Table 1: Performance evaluation of various Fusion Algorithms

\begin{tabular}{|l|c|c|c|}
\hline \multicolumn{1}{|c|}{ Fusion Method } & MI & $\mathbf{Q}^{\mathbf{A B} / \mathbf{F}}$ & Spatial Frequency \\
\hline Average & 4.9722 & 0.6629 & 09.1078 \\
\hline Select Maximum & 5.6043 & 0.5793 & 09.2172 \\
\hline Select Minimum & 5.9159 & 0.7340 & 12.4169 \\
\hline Laplacian Pyramid & 4.5623 & 0.8041 & 16.9250 \\
\hline Gradient Pyramid & 3.9768 & 0.7570 & 13.7894 \\
\hline DWT & 4.1178 & 0.7930 & 16.7954 \\
\hline Bilateral Gradient & 5.3671 & 0.8020 & 16.9230 \\
\hline Laplacian Pyramid +Bilateral Gradient & 6.4671 & 0.8302 & 17.6230 \\
\hline DWT + Bilateral Gradient & 6.6671 & 0.8620 & 17.9250 \\
\hline Dual Tree Complex Wavelet Transform & 7.1430 & 0.9010 & 18.1320 \\
\hline
\end{tabular}

Table 2: Performance evaluation of various Fusion Algorithms ( $\sigma$ vs PSNR and SSIM )

\begin{tabular}{|c|c|c|c|}
\hline$\sigma$ & DWT & $\begin{array}{c}\text { DWT +Bilateral } \\
\text { Gradient }\end{array}$ & DTCDWT \\
\hline \multirow{2}{*}{10} & 40.7806 & 44.0166 & 45.9238 \\
& 0.6944 & 0.8024 & 0.8475 \\
\hline \multirow{2}{*}{20} & 38.5817 & 41.3559 & 42.0135 \\
& 0.5980 & 0.6982 & 0.7387 \\
\hline \multirow{2}{*}{30} & 37.3448 & 40.0319 & 40.3134 \\
& 0.5483 & 0.6410 & 0.6508 \\
\hline \multirow{2}{*}{40} & 36.4721 & 37.1270 & 39.4280 \\
& 0.5163 & 0.6038 & 0.6277 \\
\hline
\end{tabular}




\section{INPUT IMAGES}
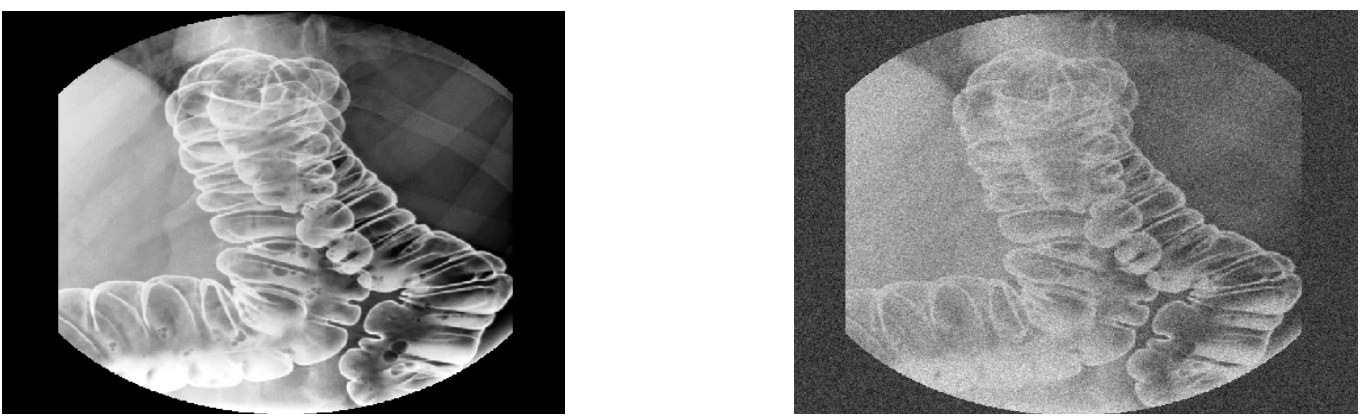

Figure 14: Input Images (a) Original Image (b) Image corrupted with Gaussian Noise ( $\sigma=30$ )
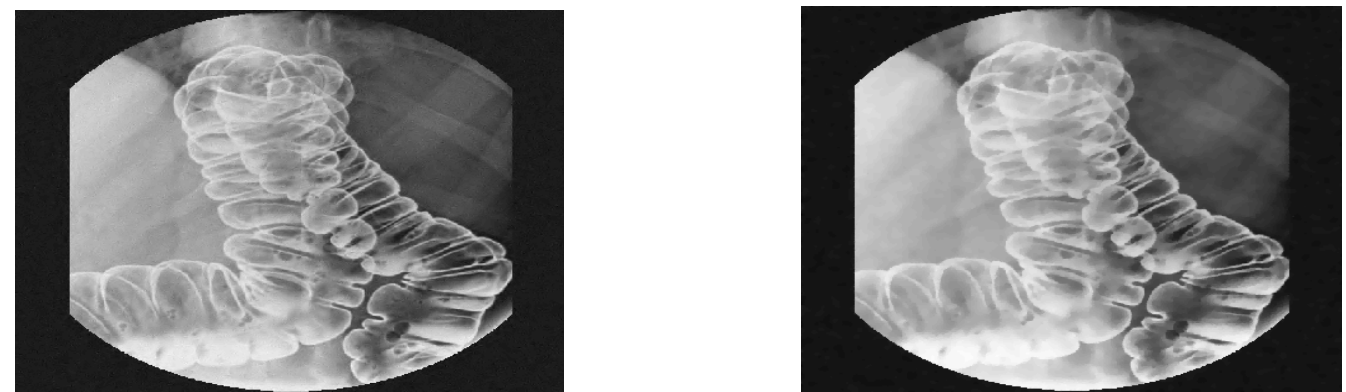

Figure 15: (a) Denoised Image Using DTCWT (b) Denoised Image Using Total Variation

\section{OUTPUT IMAGES}
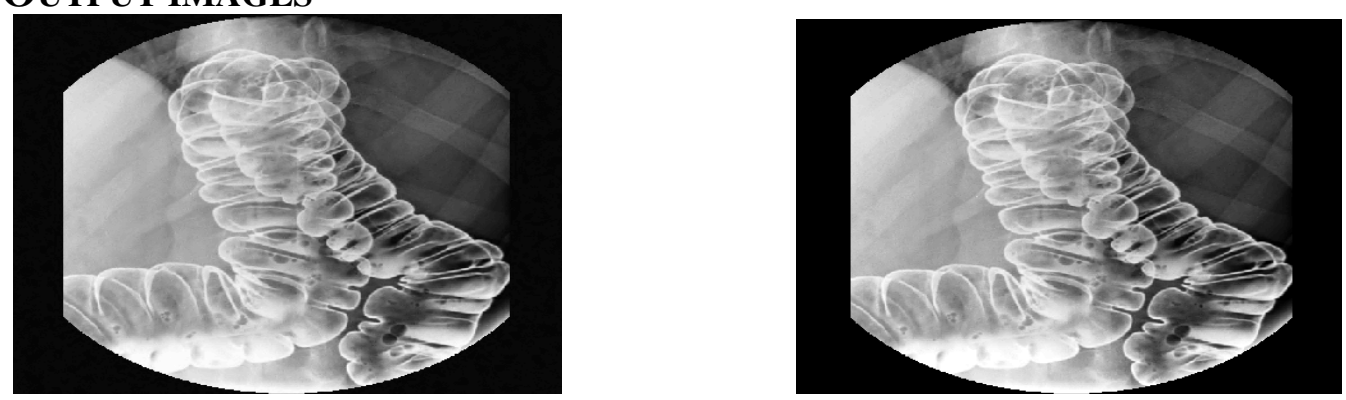

Figure 16: (a) Fused Image using DWT + Bilateral Gradient (b) Fused image using DTCWT

\section{CONCLUSION AND FUTURE WORK}

The results obtained using the above procedure is compared with the existing denoised methods. From the recorded results it is observed that the fusion of the denoised images obtained using total variational approach and Dual Tree Complex Wavelet Transform is giving better appearance and PSNR values than the input denoised images. So the denoising performance of the proposed method can be improved by choosing the better denoising procedures to denoise the images and better fusion techniques to combine the best features from these denoised images to produce the image with high perceptual quality and preservation of diagnostically relevant information. In this it is found that the fusion process by dual tree complex wavelet transform is outperforming than the existing fusion techniques. 
Signal \& Image Processing : An International Journal (SIPIJ) Vol.3, No.4, August 2012

The performance still improved by improving the denoising techniques and using directional oriented (Geometrical) multiresolution transforms such as steerable pyramid, contour lets etc for the fusion process to improve the quality of denoised images.

\section{REFERENCES}

[1] Rangaraj M. Rangayyan, "Biomedical Signal Analysis A Case study Approach”, IEEE Press, 2005.

[2] Yang Wang and Haomin Zhou, "Total Variation Wavelet-Based Medical Image Denoising", International Journal of Biomedical Imaging, Hindawi Publication, Volume 2006 Article ID89095, pages 1-6, 2006.

[3] A.Buades, B.Coll,J. M.Morel“A Review of Image Denoising Algorithms, with a new one”,Journal of Multiscale Modeling and Simulation, Vol.4, No.2, pp. 490-530, 2005.

[4] Stephane Mallat, “A Wavelet Tour of signal Processing”, Elsevier, 2006.

[6] D L Donoho and M. Jhonstone, “Wavelet shrinkage: Asymptopia?”, J.Roy.Stat.Soc., SerB,Vol.57, pp. 301-369, 1995.

[7] Imola K. Fodor, Chandrika Kamath, "Denoising through wavlet shrinkage: An empirical study", Center for applied computing Lawrence Livermore National Laboratory, July 27, 2001.

[8] David L. Donoho and Iain M. Johnstone., "Adapting to unknown smoothness via wavelet shrinkage”, Journal of the American Statistical Association, vol.90, no432, pp.1200-1224, December 1995. National Laboratory, July 27, 2001.

[9] I.Daubechies, “Ten Lectures on Wavelets”, SIAM Publishers, 1992.

[10] Z. Wang, A. C. Bovik, H. R. Sheikh and E. P. Simoncelli, "Image quality assessment: From error visibility to structural similarity," IEEE Transactions on Image Processing, vol.13, no. 4, pp. 600-612, Apr. 2004.

[11] V N Prudhvi Raj, Dr T Venkateswarlu, "ECG Signal denoising using undecimated wavelet transfrom”, accepted, ICECT2011, Kanyakumari, India.

[12] Ivan W.Selesnick, Richard G.Baraniuk, Nick G. Kingsbury, "The Dual Tree Complex Wavelet Transform”, IEEE Signal Processing Magazine, November 2005.

[13] H.B. Mitchell, Image fusion: theories, techniques and applications, Springer, 2010.

[14] W. Huang, Z. Jing, "Evaluation of focus measures in multi-focus image fusion" Pattern recognition Letters 28 (Apr.2007) 493.

[15] Chong-Yaw Wee, Measure of image sharpness using Eigenvalues, Information Sciences an International Journal, 177(2007), 2533-2552.

[16] G. Pajares, J.M. Cruz, A wavelet-based image fusion tutorial, Pattern Recognition vol. 37 (Sept. 2004) 1855.

[17] Shutao Li, Bin Yang, Multifocus image fusion by combining curvelet and wavelet transform, Pattern Recognition Letters 29(2008) 1295-1301.

[18] Jing Tian, Li Chen, Multi-Focus image fusion using wavelet statistics, Proceedings of IEEE 17th conference on Image Processing, September 26-29, Hongkong, 1205-1208. 
Signal \& Image Processing : An International Journal (SIPIJ) Vol.3, No.4, August 2012

[19] S. Arivazhagan, L. Ganesan, T.G. Subash Kumar,A modified statistical approach for image fusion using wavelet transform Signal, Image and Video Processing 3 (Jun.009) 137.

[20] R. Hassen, Z. Wang, M. Salama, Multifocus image fusion using local phase coherence measurement, Proc. Int. Conf. on Image Analysis and Recognition, Jul.2009, p. 54.

[21] Jing Tian, Li chen, Multifocus image fusion using a bilateral gradient based sharpness criterion, Optics communications 284 (2011), 80-87.

[22] Alexander Wong, William bishop, Efficient least squares fusion of MRI and CT images using a phase congruency model, Pattern Recognition letters 29 (2008), 173-180.

[23] Yingiie Zhang, Liling Ge, Efficient fusion scheme for multi-focus images by using blurring measure, Digial Signal Processing 19 (2009), 186-193.

[24] V. Aslantas, R. Kurban, Evaluation of criterion functions for the fusion of Multifocus noisy images, Proc. Int. Conf. on Signal Processing and Communications Applications, Apr. 2009, p. 492.

[25] Shutao Li, James $\mathrm{T}$ kwok, Combination of images with diverse focuses using the spatial frequency, Information Fusion 2 (2001), 169-176.

[26] P.W. Huang, C.-I. Chen, P.-L. Lin, Multi-focus image fusion based on salient edge information within adaptive focus- measuring windows, Proc. Int. Conf. on Systems, Man and Cybernetics, Oct. 2009, p. 2589. 